Revue d'histoire de l'Amérique française

ZBS REVUE D.HISTOIRE DE L'AMÉRIQUE FRANÇAISE

\title{
L'histoire du Canada de F.-X. Garneau et la critique
}

\section{Louis-Ph. Saint-Martin}

Volume 8, numéro 3, décembre 1954

URI : https://id.erudit.org/iderudit/301665ar

DOI : https://doi.org/10.7202/301665ar

Aller au sommaire du numéro

Éditeur(s)

Institut d'histoire de l'Amérique française

ISSN

0035-2357 (imprimé)

1492-1383 (numérique)

Découvrir la revue

Citer cet article

Saint-Martin, L.-P. (1954). L'histoire du Canada de F.-X. Garneau et la critique. Revue d'histoire de l'Amérique française, 8(3), 380-394.

https://doi.org/10.7202/301665ar d'utilisation que vous pouvez consulter en ligne.

https://apropos.erudit.org/fr/usagers/politique-dutilisation/ 


\section{L'HISTOIRE DU CANADA DE F.-X. GARNEAU ET LA CRITIQUE}

"Au mois d'août de 1845, Québec s'extasia de voir à la vitrine de la librairie Crémazie, rue de la Fabrique, entre des livres de Paris, un fort volume in-octavo à couverture grise, qui portait ce titre: Histoire du Canada depuis sa découverte jusqu'à nos jours. Par François-Xavier Garneau. Ce fut l'événement littéraire de l'année et même de la période. L'ouvrage produisit l'effet d'une révélation. L'histoire canadienne apparaissait pour la première fois dans toute sa gloire héroïque de croisade française et catholique au milieu des forêts du nouveau monde."'

Malgré cette appréciation si élogieuse, on peut dire que l'ouvrage fut diversement accueilli selon les milieux où il fut reçu. Il fut tantôt couvert d'éloges, tantôt vertement critiqué, et cela tant dans les journaux canadiens que dans les revues françaises. Faire l'historique de ces diverses critiques, analyser leur contenu et juger leur valeur, tel est le sujet de la présente étude.

La première appréciation de l'Histoire du Canada parut dans la Minerve de Montréal, le premier septembre 1845. L'auteur anonyme, après avoir analysé l'ouvrage et en avoir donné un résumé substantiel, appréciait ainsi le mérite de l'historien: "Nous avons lu tout le volume presque d'une haleine et avec un entrainement inexprimable. Le style est partout sans reproche. ... L'auteur, dans un magnifique discours préliminaire, développe au lecteur ses idées sur l'histoire et signale en passant la révolution qu'elle a subie depuis la découverte d'un nouvel hémisphère. Cet ouvrage formera une époque remarquable dans les annales de la littérature canadienne, et va opérer une révolution étonnante dans les dispositions des jeunes gens. La jeunesse canadienne va savourer un ouvrage où elle trouvera en même temps l'agréable et l'utile, la beauté du style et

1. G. Lanctot, Garneau, bistorien national (Montréal, 1946), 51. 
la connaissance des faits. . . . Le mérite d'un écrivain qui trouve le moyen d'intéresser si vivement par le récit de faits aussi peu remarquables que ceux du commencement de l'histoire de cette colonie, est assurément incalculable. Il faut avoir un talent supérieur, pour pouvoir varier et éviter la monotonie de la narration dans une histoire où les évènements sont presque tous ressemblants. ... Si les autres volumes ne diffèrent point du premier, nous pourrons nous flatter de posséder une histoire parfaite et sans reproche de notre pays."

Cette critique, on le voit facilement, n'était que louange et ne formulait aucune réserve. Tout était sans reproche. Un autre son de cloche allait se faire entendre dans le Canadien, de Québec, le vingt-et-un novembre de la même année 1845 . Cette nouvelle appréciation, assez sympathique dans l'ensemble, émettait cependant des réserves sur bien des points. L'auteur, caché sous le pseudonyme T.P.S., louait d'abord la valeur littéraire de l'ouvrage. "Ce livre, disait-il, mérite une place distinguée dans notre littérature. La rapidité de la narration, jointe à la clarté du style, fixe l'attention du lecteur. Les faits s'y rattachent sans effort les uns aux autres."

Dans sa critique du fond, T.P.S. s'arrêtait surtout à juger l'attitude de Garneau sur l'exclusion des Huguenots de la Nouvelle-France et sur les difficultés qu'avait entraînées la querelle au sujet de la traite de l'eaude-vie. Pour condamner la première attitude de l'historien, l'auteur raisonnait ainsi : "Si M. Garneau aime le Canada, ne doit-il pas aimer cette population toute catholique, remplie d'une foi, d'une piété, d'une moralité que tous les étrangers admirent? ne doit-il pas aimer ces établissements précieux que le catholicisme seul sait fonder et maintenir et auxquels il rend lui-même un si éclatant et si juste hommage ?" Après avoir cité un passage où Garneau affirmait que l'expérience n'avait pas encore appris que l'intérêt religieux même exigeait impérieusement que tout fût sacrifié à l'avancement et à la consolidation des colonies, T.P.S. le commentait ainsi: "Il fallait tout sacrifier! tout, même la religion et la morale ? Pour ma part, je suis bien persuadé qu'une semblable pensée est loin du coeur et de l'esprit de M. Garneau; je regretterai toujours qu'une expression aussi forte que celle-là soit sortie de la plume d'un auteur que je voudrais voir sans reproche. . . Je comprends son erreur. Il croit que son pays aurait été plus riche en industries de toutes espèces : douce illusion qui fait honneur à son coeur patriotique, et que tout $\mathrm{Ca}$ - 
nadien lui pardonnera en faveur de son motif. . . Je me suis dit plusieurs fois à moi-même: "L'auteur serait-il un de ces hommes pour qui la religion n'est quelque chose qu'autant qu'elle peut servir les intérêts matériels du peuple? Croit-il à la vérité de la religion catholique ? Ces questions que je me suis faites plusieurs fois à moi-même, d'autres me les ont adressées et j'avoue que je n'ai pu répondre à une accusation que ses compatriotes regardent avec raison comme très grave et qui a déjà été signalée par l'habile correspondant du Courrier des Etats-Unis". Ne sent-on pas sous ces critiques directes et judicieuses le désir de ne pas "verser dans la critique amère" et d'excuser le plus possible les torts de l'historien?

Au sujet des luttes qui marquèrent les relations entre le gouverneur de la colonie et l'évêque de Québec sur la question de l'eau-de-vie, le critique n'est pas moins insinuant. Garneau avait écrit: "Les gouverneurs pieux sont blâmés pour leur faiblesse et leur asservissement à un zèle religieux qui mettait le gouvernement à la discrétion du clergé." 2 Voici comment T.P.S. lui répond: "Quoi, rendre des ordonnances sur les représentations des missionnaires, c'est leur assujettir un pouvoir qui doit rester indépendant!' Il rapporte une longue phrase où l'historien avait exprimé des idées peu favorables à la religion, et il la commenté en ces termes: "Je ne sais si je me trompe, mais il me semble que le premier membre signifie: "Si on se fut querellé pour des intérêts matériels, je me serais uni de bon coeur aux combattants, mais parce qu'on ne s'est disputé que pour des fins religieuses, je ne puis que blâmer les deux partis." La religion en vaut si peu la peine ! A la bonne heure, qu'on se querelle pour une amélioration politique et matérielle: ces intérêts valent bien la peine qu'on se les dispute, qu'on traverse les mers pour les soutenir, qu'on use de toute son influence! Comme Canadien, je proteste de bon coeur contre de semblables idées." En terminant sa critique, l'auteur s'adressait directement à l'historien et lui disait: "Continuez vos recherches, donnez aux volumes que nous attendons le même intérêt qu'au premier; mais souvenez-vous du rapport intime que vous avez vousmême affirmé exister entre notre religion et notre nationalité. Toute ma crainte, c'est que le religieux peuple canadien ne vous pardonne que difficilement d'avoir été puiser vos inspirations dans des ouvrages re-

2. Histoire du Canada, I : 284. 
gardés généralement comme hostiles au catholicisme. L'historien peut et doit blâmer tout excès, de quelque rang qu'il vienne, mais qu'il se rappelle que l'abus ne détruit nul droit, n'infirme aucune autorité, et que si deux intérêts se trouvent en contact, il faut faire pencher la balance en faveur du plus grand et du plus nécessaire."

Un autre critique, caché lui aussi sous un pseudonyme, " $\mathrm{Y}$ ", se montre aussi sévère dans le même journal, Le Canadien, le douze décembre 1845. Il analyse d'abord la conception de l'histoire chez Garneau et il étudie les sources auxquelles il a puisé. "En lisant la nouvelle histoire, écrit-il, nous nous sommes cru vingt fois transporté aux cours de la Sorbonne ou du collège de France. La marche rapide de l'écrivain, l'inyestigation prétentieuse des moindres faits, le style frondeur et pompeux, la teinte continue d'antipathie contre l'esprit religieux et surtout l'esprit catholique, les doléances ou les récriminations les plus acerbes touchant certaines questions brûlantes, telles que la tolérance, le gouvernement ecclésiastique, l'autorité des souverains, la "grande figure du peuple" .... tous ces caractères, disons-nous, nous rappelaient bon gré mal gré, le "savant et ingénieux" M. Michelet, que l'auteur avoue d'ailleurs avoir pris pour guide plus d'une fois."

Puis le critique rappelle les idées de Garneau sur le peuple méconnu par les grands, sur l'obscurantisme du Moyen Age. Au sujet de l'oeuvre humanitaire de l'Eglise, il pose ensuite à l'historien une question qu'il juge lui-même embarrassante: "Un embarras sérieux pour notre historien serait de l'engager à expliquer publiquement au peuple canadien, lui qui doit sa civilisation, non au philosophisme, mais à la religion, non aux historiens courtisans, mais aux jésuites et aux capucins, ce que c'est que la civilisation sans christianisme. . . . L'embarras deviendrait encore plus scabreux, s'il fallait prouver au peuple canadien que le christianisme a des tendances humanitaires; c'est-à-dire, en laissant de côté le jargon philosophique, qu'il est bon à quelque chose dans l'intérêt de l'humanité."

Dans la conclusion de son article, l'auteur condense non seulement l'esprit de sa critique, mais l'esprit même de l'ouvrage historique qu'il étudie." "Malgré les qualités purement littéraires qui distinguent l'Histoire du Canada; malgré les vues patriotiques et louables de son estimable auteur, l'esprit qui a dicté cette oeuvre et qui règne d'un bout à l'autre du volume. . . la rend suspecte à tout Canadien religieux et national; car 
notre salut politique comme notre salut spirituel a sa condition sine qua non dans le catholicisme, mais uniquement dans le catholicisme de Notre Saint Père le Pape; pas d'autre : celui de Michelet et consorts n'ayant que des tendances humanitaires, ou étant un culte mort, ne nous convient pas plus qu'à la noble Suisse et à la malheureuse Irlande. Oui, certes, nous ne serons véritablement Canadiens que tant que nous resterons véritablement catholiques. En outre, il appartiendra aux instituteurs de la jeunesse, aux chefs responsables de notre haut enseignement, à peser dans leur sagesse si la nouvelle histoire, supposé qu'elle ne se réforme pas, peut avoir entrée libre dans nos collèges. Enfin, et nous le disons avec peine, l'esprit que nous signalons dans l'Histoire du Canada ternit une belle oeuvre. . . Ce que l'auteur actuel n'a pas atteint, qui l'empêche de le conquérir par une noble et courageuse revue de son oeuvre ? Car nous n'hésiterons pas à le dire, : à son talent appartient la tâche qu'il s'est imposée. Seulement qu'il puise à d'autres sources." Cette étude, dans son ensemble, contient donc certains éloges bien mérités, mais surtout une critique "franche, loyale, impartiale et fidèle" des principaux problèmes qui ont attiré l'attention de l'auteur.

Dans un autre article publié encore dans le Canadien, le quatre mars 1846, le même auteur, encouragé par de nombreuses lettres de félicitations, revient à la charge et se montre plus sarcastique et plus violent. Il revient sur la querelle de l'eau-de-vie et réfute le libéralisme doctrinal et le scepticisme religieux qui imprègnent l'esprit de l'Histoire du Canada, "esprit anti-catholique et anti-canadien". Il appelle l'oeuvre "un mensonge, un méfait social et politique". Voici comment il juge de nouveau la question de la traite de l'eau-de-vie: "Rien n'est admirable dans la nouvelle Histoire du Canada comme le talent de l'auteur à confondre continuellement les deux pouvoirs qui gouvernent les sociétés chrétiennes, ou à mettre sous les pieds du pouvoir temporel l'autorité spirituelle du pouvoir ecclésiastique. . . . De ce que le zèle du digne évêque maintient envers et contre tout la détermination qu'il a prise contre les effets funestes de l'eau-de-vie, l'auteur veut qu'il y ait là empiétation du clergé, assujettissement du pouvoir civil à l'autorité spirituelle, position anormale, obligation contractée avec le sanctuaire. Si l'Etat entre en voie d'accommodement avec l'Eglise pour réprimer les désordres, alors "le gouvernement, tout entier à son zèle religieux, oublie qu'en se mettant ainsi à la discrétion du clergé, il ouvre la porte à mille difficultés". Il n'est pas difficile avec des raisons de cette nature de trouver un esprit de do- 
mination au bénéfice d'un évêque et d'une cause qui, il est vrai, ne prennent jamais couleur de rose sous la plume de notre auteur."

Sur le libéralisme doctrinal et le scepticisme religieux, le critique exprime ensuite des idées qui réfutent à l'avance les vagues d'esprit librepenseur dont on sera ensuite périodiquement inondé et par lesquelles certains esprits forts (ou faibles ?) reprocheront à l'Eglise d'exercer son influence dans des domaines qui ne sont pas purement temporels, mais où la vie morale du peuple se trouve fortement engagée. "Ah! philosophes, s'écrie-t-il, que vos rêves de civilisation et de prospérité sont beaux, vraiment! Et vous, peuple canadien, que n'êtes-vous sorti d'hommes puissants à boire! Que n'êtes-vous même huguenot! car c'est encore $1 \mathbf{d}$ un des regrets de votre historien. Que n'êtes-vous ce peuple avancé qui n'a d'autres maîtres que ses passions, d'autre loi que sa VOLON'TE GENERALE! Au lieu de cette renommée religieuse, morale et heureuse, qui vous rend célèbre par tout le monde, vous brilleriez de tout l'éclat du luxe. Une autre croyance que celle que Richelieu et les dragonnades vous imposèrent vous eût appris à avoir autant de religion que de têtes. De savants professeurs-historiens, laissant héroïquement de côté tous les objets les plus chers et les plus sacrés d'une vive et légitime affection, se seraient substitués, pour vous enseigner les règles éternelles des moeurs, les arts et les sciences, à ces religieux mal appris dont l'ambition incroyable allait jusqu'à se faire casser la tête pour vous donner la lumière et la vie. Sans cela des académies, des universités brillantes vous eussent déjà rendus moins crédules à l'endroit de vos idées et de vos affections religieuses, vous eussent fait prendre de larges coudées dans le domaine indépendant de l'esprit humain . . . L'industrie vous inscrirait aujourd'hui avec gloire parmi les nations éclairées du globe. . . Avec un peu plus de vices, il est vrai, avec l'inconvénient de vivre sans foi ou avec une foi bâtie par la raison : toutefois, pour compenser amplement tout cela, vous auriez un nom et des vertus dignes de l'époque." Ne retrouve-t-on pas ici les accusations actuelles d'obscurantisme, d'influence rétrograde de l'Eglise et de ses institutions?

L'auteur invite alors la jeunesse à se garantir contre ces idées, à ne pas croire sur parole, à se prémunir contre l'engouement pour des oeuvres pompeuses, originales, soi-disant philosophiques. Puis il cite le P. Lacordaire qui réprouve la sagesse de ceux qui osent se poser sans crainte 
devant le Christ et son Eglise pour les mettre au ban de la raison humaine. Et il conclut par ce jugement général: "Les qualités, toutes de forme, ne rachètent en rien les étranges principes qui lui servent de fond; . . . votre oeuvre doit rencontrer la réprobation de tout Canadien qui sait mettre au premier rang dans ses affections, sa religion et tous les principes d'ordre et de sage liberté qu'il a reçus de ses pères."

La Minerve du vingt-huit décembre 1883 reproduit une lettre de Pierre Margry, adressée en février 1846 au Docteur Leprohon, de Montréal. Margry y loue l'Histoire de Garneau, qu'il trouve bien supérieure à celle de Charlevoix. "Il y a, écrit-il, dans l'ouvrage de M. Garneau, de la méthode, on y voit nettement les choses, tandis que dans Charlevoix, la diffusion, la confusion qui y règnent, ne permettent pas même d'observer ce qu'il contient. . M. Garneau a creusé davantage et ses jugements me semblent pour la plupart bons; du moins, j'ai eu l'occasion de me rencontrer avec lui dans la plupart des cas. . . Le livre de M. Garneau est pour moi l'oeuvre d'un homme de talent. Les critiques amères du Canadien - citées plus haut - me font même ajouter, oeuvre d'un homme de courage." Par cet article, Margry se range donc au nombre de ceux qui ont loué Garneau sans réserve et qui ont trouvé son oeuvre "sans reproche".

Dans une longue étude publiée le 15 juillet 1853 dans la Revue des Deux-Mondes et consacrée à l'Histoire du Canada de Garneau, M. Théodore Pavie considérait surtout le problème de la colonisation en Nouvelle-France et s'appliquait à montrer le sens colonisateur de la France. Quelques brefs passages étaient consacrés à l'appréciation de l'oeuvre historique elle-même. L'auteur écrivait entre autres choses: "M. Garneau a gardé le culte d'un glorieux passé, et il se montre le champion du parti français. Il lutte avec énergie et conviction en faveur des libertés que ses pères ont sauvées du naufrage de leur nationalité, et cette noble cause, il la défend avec l'énergie d'un Canadien de vieille souche." A peine trouve-t-on dans cet article quelques observations critiques: "Il y a peutêtre à la surface de ses idées une certaine ébullition, une ardeur gauloise qui va jusqu'à l'entraînement; il a lu beaucoup, et ses citations feraient supposer qu'il n'est pas assez en garde contre l'exagération et l'emphase de certains écrivains déclamatoires, Raynal par exemple. Cependant, sur les questions fondamentales, il a des jugements solides et empreints d'impartialité. Ce qui lui donne une véritable importance, c'est l'abondance 
des documents qu'il renferme... C'est une belle et grande page de notre histoire." 3

En 1854, dans le Correspondant de Paris, M. Louis-Ignace Moreau livrait au public en même temps qu'un bel éloge de notre historien, une solide critique des principales erreurs de Garneau. Laissons l'auteur exposer lui-même le but de son étude: "Nous voulons examiner l'Histoire $d u$ Canada à ce point de vue, parce que nous y trouvons l'occasion de montrer quelles furent les pensées et les espérances de la France dans la fondation de ses établissements coloniaux sur le continent américain. On ne sait plus assez combien elle a mérité, par son zèle à appeler les races indiennes à la lumière de l'Evangile, son glorieux surnom de nation très chrétienne." 4 Pour lui, Garneau n'a pas suffisamment mis en lumière les buts religieux de la France dans son oeuvre colonisatrice. Il estime que la fille aînée de l'Eglise visait d'abord à la conversion des infidèles, et que les intérêts matériels passaient au second plan.

Il attaque ensuite de front certains faux principes de l'historien: "Il y avait, écrit-il, des inconvénients contre lesquels il ne s'est pas mis en garde et des périls qu'il n'a pas su éviter. Il a reçu ses doctrines, ses idées, ses opinions des Américains. Il pense comme eux de l'origine des sociétés, des devoirs des gouvernements, de la liberté des citoyens, de l'indépendance des hommes. Comme eux encore, il n'écarte pas seulement de la direction des peuples toute action de la religion et des prêtres; il la condamne, il la réprouve; il s'en irrite. Catholique, sa philosophie n'est pas chrétienne. Il a contre les ministres du culte dont il fait profession, les préjugés de l'hérésie et de l'incrédulité. Et c'est avec ces préjugés le plus souvent qu'il juge leur conduite dans la part qu'ils ont dû prendre dans le développement de la colonie." 5

Dans la querelle sur la traite de l'eau-de-vie, M. Moreau prend franchement parti contre M. Garneau. "Le clergé, dit-il, en vint jusqu'à fulminer une sentence d'excommunication contre tous ceux qui transgresseraient ses défenses. Il y eut de longues luttes entre le pouvoir spirituel et le gouvernement temporel de la colonie. M. Garneau prend parti pour ce dernier. S'il ne donne pas au clergé tous les torts, il l'accuse

3. Revue des Deux Mondes (Paris, 1853), 302.

4. Le Correspondant (Paris, 1854), 347.

5. Ibid., 346. 
au fond d'avoir été mû par un désir ardent de domination." 6 Voici les raisons que Moreau apporte en faveur de son attitude: "On éteignait lentement la vie de la nature chez des hommes qu'ils (les missionnaires) s'efforçaient de faire naître à la vie de la grâce. Il ne leur était pas permis de le souffrir silencieusement... sans manquer à tous les devoirs de leur ministère... Ces raisons sont-elles d'un ordre trop élevé ? Selon lui, la traite de l'eau-de-vie était nécessaire au commerce des pelleteries."' Morean montre que d'ailleurs les intérêts de la colonie n'y trouvaient guère leur profit, puisque les traitants étaient de mauvais colons. Puis il fait voir dans l'attitude des missionnaires des raisons de devoir, d'utilité dans le présent, de prévoyance pour l'avenir. "Les missionnaires préparaient l'avènement d'une nationalité d'autant plus forte qu'elle serait plus profondément imprégnée de la foi catholique." 8

L'une des plus belles pages de cet article, c'est sans contredit celle où l'auteur fait l'éloge du système d'enseignement du Canada français. Il y montre la légitimité de notre enseignement confessionnel, enseignement que ne peuvent adopter les protestants, puisqu'ils sont divisés en une infinité de sectes et qu'ils ne peuvent s'entendre pour établir les bases d'un solide enseignement religieux. Puis il conclut par ce bel éloge du rôle du clergé dans la vie du peuple canadien: "Les Canadiens doivent au clergé de garder encore aujourd'hui ce qui seul les distingue des étrangers qui les enveloppent, qui les pressent, qui voudraient les absorber : leur religion et leur langue... Il y a donc dans le reproche de $\mathbf{M}$. Garneau de l'injustice et de l'ingratitude."

De la forme littéraire de l'ouvrage, $M$. Moreau ne disait en somme que du bien: "C'est la langue française du XVIIe siècle... elle est en général simple et correcte, si ce n'est qu'il s'y mêle parfois une sorte d'archaïsme qui n'est pourtant pas sans charme; mais elle a plus de nerf que d'élégance. Elle a d'ailleurs la clarté, la gravité, la précision qui conviennent à l'histoire."

En cette même année 1854, Maximilien Bibaud, fils de Michel, qui avait écrit en 1837 et en 1844 une Histoire du Canada en deux tomes, pu-
6. Ibid., 362 .
7. Ibid., 363.
8. Ibid., 365 .
9. Ibid., 368 . 
blia une plaquette intitulée: Revue critique de l'Histoire du Canada de $M$. Garneau, à laquelle il donna comme sous-titre: "Le charlatanisme dans l'histoire". L'appréciation que Bibaud fait de Garneau est d'une insolence extrême, d'une injustice inexplicable. Voici ce qu'il écrit dans l'avis préliminaire de son opuscule: "N'ayant pas le loisir d'appliquer notre traité de la critique historique en rapport avec l'Histoire du Canada de M. Garneau..., ni les moyens de publier cet ouvrage, nous livrons au public le présent opuscule, dans lequel nous avançons et nous prouvons qu'il ne peut être assigné aucun rang à l'oeuvre de ce monsieur dans la république des lettres :

1 - parce qu'elle décèle l'ignorance de plusieurs choses qu'il n'est pas permis à celui qui écrit l'histoire d'ignorer;

2 - parce qu'on n'y découvre aucune des dispositions demandées à l'historien;

3 - parce que les infidélités y sont aussi nombreuses que les erreurs;

4 - parce que la grammaire n'y est pas respectée."

Après quelques considérations générales sur la forme littéraire de l'oeuvre, où il écrit que "le langage est fort disparate", que les phrases ont "un air guindé et contraint", que "les mots sont rarement employés dans leur véritable acception", que "l'ouvrage, où les anglicismes les moins pardonnables fourmillent, est composé en partie de fragments traduits de l'anglais servilement et barbarement", que "la phraséologie n'est point supportable", "les paragraphes sont mal distribués, la ponctuation n'est pas passable, l'orthographe n'est pas même observée"10, après cela, dis-je, le critique passe en revue les principales fautes de langage, les erreurs concernant le droit public et privé, les erreurs au sujet des intendants, les infidélités de l'auteur, les hypothèses dénuées de preuves, les contradictions et les autres bévues, les erreurs sur l'histoire générale, etc.

Dans son dernier article, il juge l'esprit du livre d'une manière franchement injuste et exagérée. "M. Garneau, écrit-il, est philosophaste, mais de quelle secte, nous ne savons, car il mêle ensemble toutes les doctrines ... Il s'est montré catholique en "un" endroit . . . Il a insulté sa religion aussi souvent qu'il a pu."' Malgré son esprit sceptique et sou-

10. Maximilien Bibaud, Revue critique de l'Histoire du Canada de M. Garneau (Montréal, 1855), 6-7.

11. Ibid., 43-45. 
vent libre-penseur, on ne saurait porter sur Garneau un jugement aussi sévère, car on trouve dans son histoire bien des passages où il porte des jugements empreints d'esprit chrétien.

Bibaud résume ainsi son opinion sur l'ouvrage de Garneau : "Il est une de ces publications hasardées qui, d'une fois, décréditent un homme qui se met à écrire... Il a passé pour un chef-d'oeuvre, mais c'est là un fait humiliant dans les annales canadiennes." 12 Cette brochure de Bibaud contient des critiques injustes, outrées, inspirées par le parti-pris et un secret antagonisme. Mais elle contient aussi des remarques vraies et judicieuses sur certaines erreurs, d'ailleurs inévitables dans un ouvıage de ce genre, et dont Garneau a sûrement profité dans ses autres éditions.

Que peut-on conclure de critiques si nombreuses et si diverses?

Pour ce qui est du mérite littéraire de l'oeuvre de Garneau, "ses critiques, comme ses admirateurs, en ont reconnu la vaste conception, l'ordonnance habile et la riche exécution". 13 "A tout prendre, sa prose reste une bonne prose, forte et pure, droite et loyale, qui retarde quelque peu, mais qui fleure bon la vieille province française. Jugée à la mesure de la prose contemporaine, elle se place au premier rang par son ordonnance logique, sa maîtrise de la période, sa robustesse et sa démarche entraînante." 14 "Le style est à la hauteur de la pensée, et révèle un écrivain d'élite. Il a de l'ampleur, de la précision et de l'éclat; mais il est surtout remarquable par la verve et l'énergie... Il se distingue par une qualité qui fait son véritable mérite et qu'explique l'inspiration sous laquelle l'auteur a écrit. C'est dans un élan d'enthousiasme patriotique, de fierté nationale blessée, qu'il a conçu la pensée de son livre, que sa vocation d'historien lui est apparue. Ce sentiment, qui s'exaltait à mesure qu'il écrivait, a empreint son style d'une beauté mâle, d'une ardeur de conviction, d'une chaleur et d'une vivacité d'expression qui entraînent et passionnent surtout le lecteur canadien. On sent partout que le frisson du patriotisme a passé sur ces pages." 15

12. Ibid., 7-8.

13. H.-R. Casgrain, De Gaspé et Garneau (Montréal, 1912), 133.

14. G. Lanctot, Garneau, bistorien national (Montréal, 1946) 173.

15. H.-R. Casgrain, De Gaspé à Garneau (Montréal, 1912), 138. 
Les critiques se sont plu aussi à louer la méthode historique suivie par Garneau. "Le premier de nos historiens à suivre une discipline scientifique, écrit $M$. Gustave Lanctot, Garneau s'élève du coup au-dessus de tous ses devanciers, la plupart simples annalistes et chronologues. Sur la plupart d'entre eux aussi, il possède une autre supériorité : il sait ordonner sa matière. Où ses devanciers suivent servilement la succession des événements et s'embourbent dans le sable des incidents, Garneau a construit un ensemble historique aux belles proportions et d'une logique ordonnance. Prompt à s'élever au-dessus des faits pour en dégager les causes et les conséquences, son esprit excelle à les grouper autour d'une idéemère ou d'un événement capital. En une série de chapitres, logiquement distribués, il dresse ainsi des tableaux successifs des époques et des institutions, qui permettent à l'esprit de suivre facilement les étapes de l'histoire canadienne et de mieux pénétrer le caractère de son évolution."16

Le but même de son oeuvre, Garneau l'a bien mis en lumière dans une lettre qu'il adressait le 19 mai 1846 à Lord Elgin, gouverneur général du Canada. Personne n'a osé le contester. "J'ai entrepris ce travail, écrit-il, dans le but de rétablir la vérité, si souvent défigurée, et de repousser les attaques et les insultes dont mes compatriotes ont été et sont encore journellement l'objet de la part d'hommes qui voudraient les opprimer et les exploiter tout à la fois." 17

Quant aux idées erronées que Garneau a exprimées dans son ouvrage, si elles ne peuvent être excusées, elles peuvent au moins s'expliquer. "Le grand malheur de F.-X. Garneau a été, non pas tant d'avoir reçu ses doctrines des Américains - comme l'écrivait Moreau - mais de n'avoir pas eu de formation classique chrétienne. Sans préparation suffisante, il a abordé des auteurs dangereux et quelquefois pervers."। "Il appartient à la grande école d'Augustin Thierry, dont il était l'admirateur passionné : il en a les qualités et même les défauts, la manière large, le regard philosophique, et quelque chose de son talent dramatique et littéraire; mais aussi il en a les tendances rationalistes et les préjugés politiques. Ce fut le malheur de son éducation solitaire, abandonnée à elle-

16. G. Lanctot, op.cit., 154.

17. H.-R. Gasgrain, op.cit., 108-109.

18. G. Robitaille, "L'oeuvre de Garneau et la critique de son temps", Centenaire de l'Histoire du Canada de F.-X. Garneau. Deuxième semaine d'Histoire à l'Université de Montréal, 23-27 avril 1945 (Société historique de Montréal, 1945), 141. 
même, privée de cette salutaire direction qu'impriment aux jeunes talents nos grandes institutions religieuses." 19

Certaines idées erronées s'expliquent aussi par la fin, toujours noble, qui guidait la pensée et les actes de l'historien. S'en surprendra qui voudra, mais il semble qu'au moins on ne puisse douter des intentions. La grande liberté de son esprit, il la manifeste dans une lettre écrite à son ami Chauveau. "Le respect, dit-il, que j'ai toujours eu pour mes convictions et pour l'indépendance de mes opinions, en jugeant les hommes et les choses, dans mon Histoire du Canada, devait peut-être ruiner mon avenir. Mais je savais d'avance la conséquence de ma conduite. Puisque j'ai fait pareil sacrifice, qui peut atteindre mes enfants, j'étais prêt à faire le sacrifice, non moins sensible pour moi, de votre approbation." 20

$\mathrm{Au}$ sujet de la liberté de conscience, l'une des principales idées que l'on a reprochées à Garneau, celui-ci était aussi animé des meilleures intentions. Pour nous en convaincre, écoutons ce qu'il écrivait à son critique du Correspondant de Paris, M. Moreau. "La suite des événements vous aurait fait voir que ce n'était pas sans de graves motifs que j'avais adopté dans toute sa force le principe de la liberté de conscience. En effet, sans ce principe protecteur, où les catholiques en seraient-ils dans l'Amérique du Nord avec les huit-dixièmes de la population protestante, et des gouvernements partout protestants ?... C'est aussi à l'aide de ce principe de tolérance que j'ai pu défendre les catholiques canadiens contre les attentats du gouvernement protestant de l'Angleterre après la conquête. Le blâme que j'avais porté contre le gouvernement français, donnait de la force à mes paroles, aux yeux des protestants eux-mêmes, lorsque je blâmais leur conduite depuis qu'ils étaient les maîtres, et ne laissait rien à me répondre." 21 A ce sujet, le tort de Garneau, selon certains critiques, a été, non pas tant d'affirmer le principe de tolérance, mais d'en pousser les conclusions à l'extrême.

Puisqu'il s'agit de porter un jugement sur toute la pensée de Garneau, peut-on ajouter foi au témoignage de l'abbé Casgrain, lorsqu'il écrit: "Garneau a donné une preuve éclatante de sa piété filiale envers l'Eglise en soumettant humblement la dernière édition de son Histoire à

19. H.-R. Casgrain, op.cit., 134.

20. G. Lanctot, op.cit., 160-161.

21. H.-R. Casgrain, op.cit., 106-107. 
un ecclésiastique compétent, et en faisant plein droit aux observations qui lui avaient été suggérées" ?22 Cette preuve éclatante ne perd-elle pas de son éclat, lorsqu'on considère le témoignage d'Edmond Lareau, un contemporain de Garneau ? "Afin d'avoir la paix, écrit ce critique, Garneau consentit à corriger certaines parties de son ouvrage, qui n'en est pas moins, auprès de certains esprits, entaché de gallicanisme. Il est facile de comprendre que l'historien a subi cette influence sans l'accepter volontairement. C'est afin de rendre son livre plus acceptable à la majorité des lecteurs canadiens qu'il a dû accepter les conseils, les avis et les remontrances du clergé canadien; mais le premier jet a été le fruit de sa pensée intime, l'opinion raisonnée du philosophe et du penseur, elle prévaudra." 23 Darveau écrit de son côté que Garneau a fait certaines corrections "pour se soustraire à la persécution". ${ }^{24} \mathrm{M}$. Hector Garneau luimême, le petit-fils de l'historien, n'a-t-il pas confirmé cette prétention, en prenant pour modèle de ses $5 \mathrm{e}$, 6e et $7 \mathrm{e}$ éditions, non la $3 \mathrm{e}$ corrigée par son grand-père, mais la première, et cela pour rendre justice à l'auteur qui avait sacrifié du texte à certaines pressions ?

Indépendamment du but poursuivi, peut-on dire que les corrections effectuées purifiaient totalement la pensée de l'auteur? Après avoir comparé la première et la troisième éditions de l'Histoire du Canada et avoir découvert dans chacune certaines idées osées en matière religieuse, M. Marcel Trudel tire cette conclusion: "Dans cette troisième édition, Garneau s'est appliqué à retrancher les formules voltairiennes choquantes plus qu'à christianiser le fond même de son Histoire."25

Le critique qui fera le jour sur cette importante question méritera grandement de ses compatriotes, car c'est là une question où toute la gloire de l'historien, non moins que celle de tout notre peuple, est engagée. Si la pensée de Garneau n'a jamais été corrigée, ou si elle a été corrigée à regret, mérite-t-il vraiment le titre d'historien national ? Lareau lui donne ce titre, "non seulement parce que son livre est l'apothéose impartiale des canadiens français et de la race latine en Amérique, mais encore parce qu'il réunit en lui l'idéal populaire et démocratique pris

22. Ibid., 100.

23. E. Lareau, Histoire de la littérature canadienne (Montréal, 1874), 160.

24. L.-M. Darveau, Nos bommes de lettre (1873), 90. I : 185.

25. Marcel Trudel, L'influence de Voltaire au Canada (2 vol., Montréal, 1946), 
dans une large extension". ${ }^{26}$ Ce titre d'historien national, il semble que celui qui le mérite soit celui qui a non seulement relaté la vie du peuple au milieu duquel il a vécu, mais qui a su comprendre et exprimer les deux caractères dominants de notre nationalité : ses innéités françaises et son catholicisme. En déformer un, en le tronquant, c'est présenter une fausse image de cette nationalité dont on a voulu fortifier les aspirations.

En attendant que plus de lumière se fasse sur cette grave question, conservons à Garneau ce titre glorieux que lui ont décerné ses contemporains. "C'est lui qui, le premier, a pénétré dans le chaos de nos archives et penché le flambeau de la science sur ces ténèbres... C'est lui qui, le premier, à force de patriotisme, de dévouement, de travail, de patientes recherches, de veilles qui ont usé ses jours, fané sa vie dans sa fleur, est parvenu à venger l'honneur de nos ancêtres outragés, à relever nos fronts courbés par les désastres de la conquête, en un mot, à nous révéler à nous-mêmes. Qui donc mieux que lui mériterait le titre glorieux que la voix unanime des Canadiens, ses contemporains, lui a décerné ? Nous avons donc droit de l'espérer, l'avenir s'unira au présent pour le saluer du nom d'HISTORIEN NATIONAL." 27 En se plaçant à ce point de vue, qui oserait contredire le jugement et ne pas répondre au souhait du brillant historien?

Louis-Ph. SAINT-MARTIN, ptre, L.-ès-L.

26. E. Lareau, Histoire de la littérature canadienne (Montréal, 1874), 159.

27. H.-R. Casgrain, op.cit., 139. 